\title{
Correction to: A phase II study of post-transplant cyclophosphamide combined with tacrolimus for GVHD prophylaxis after HLA-matched related/unrelated allogeneic hematopoietic stem cell transplantation
}

\author{
Hirohisa Nakamae ${ }^{1}$ (1) $\cdot$ Takahiko Nakane $^{1} \cdot$ Hiroshi Okamura $^{1} \cdot$ Hideo Koh $^{1} \cdot$ Yasuhiro Nakashima $^{1} \cdot$ Asao Hirose $^{1}$. \\ Mika Nakamae ${ }^{1}$. Mitsutaka Nishimoto ${ }^{1}$ Masatomo Kuno ${ }^{1}$. Yosuke Makuuchi ${ }^{1}$. Naonori Harada ${ }^{1}$. \\ Teruhito Takakuwa ${ }^{1} \cdot$ Masayuki Hino ${ }^{1}$
}

Published online: 1 November 2021

(c) Japanese Society of Hematology 2021

\section{Correction to: International Journal of Hematology https://doi.org/10.1007/s12185-021-03228-1}

In the original publication of the article, the "Conflict of interest" were not listed and should be as given below.

Conflict of interest $\mathrm{H}$. Nakamae reports research funding from Astellas Pharma Inc., Novartis Pharma K.K., and honoraria from Astellas Pharma Inc., Takeda Pharmaceutical Co., Ltd., Novartis Pharma K.K., Pfizer Japan Inc., Chugai Pharmaceutical Co., Ltd., Daiichi Sankyo Co., Ltd., Kyowa Kirin Co., Ltd., and Nippon Shinyaku Co., Ltd. (none of which are related to the submitted work). T. Nakane reports research funding from Janssen Pharmaceutical K.K., Pfizer Japan Inc., and honoraria from Daiichi Sankyo Co., Ltd., Novartis Pharma K.K., Astellas Pharma Inc., and Nippon Shinyaku Co., Ltd. (none of which are related to the submitted work). H. Okamura reports honoraria from Nippon Shinyaku Co., Ltd. H. Koh reports research funding from Chugai Pharmaceutical Co., Ltd., and Takeda Pharmaceutical Co., Ltd., and honoraria from Takeda Pharmaceutical Co., Ltd., and Novartis Pharma K.K. (none of which are related to the submitted work). Y. Nakashima reports research funding from Novartis Pharma K.K., and Astellas Pharma Inc., and honoraria from Novartis Pharma K.K., JCR Pharmaceuticals Co., Ltd., Pfizer Japan Inc., Chugai Pharmaceutical Co., Ltd., and Kyowa Kirin Co., Ltd. (none of which are related to the submitted work). M. Nakamae reports honoraria from
Novartis Pharma K.K., and payment (honoraria) to spouse as other financial or nonfinancial interests (Please refer to $\mathrm{H}$. Nakamae disclosure) (none of which are related to the submitted work). M. Nishimoto reports research funding from Janssen Pharmaceutical K.K., and honoraria from Kyowa Kirin Co., Ltd. (none of which are related to the submitted work). T. Takakuwa reports honoraria from Takeda Pharmaceutical Co., Ltd., Sanofi K.K., Janssen Pharmaceutical K.K., Novartis Pharma K.K., and Kyowa Kirin Co., Ltd. (none of which are related to the submitted work). M. Hino reports research funding from JCR Pharmaceuticals Co., Ltd., Pfizer Japan Inc., Kyowa Kirin Co., Ltd., Daiichi Sankyo Co., Ltd., Chugai Pharmaceutical Co., Ltd., Takeda Pharmaceutical Co., Ltd., Novartis Pharma K.K., and Astellas Pharma Inc., and honoraria from Astellas Pharma Inc., Kyowa Kirin Co., Ltd., Sanofi K.K., Takeda Pharmaceutical Co., Ltd., Chugai Pharmaceutical Co., Ltd., Nippon Shinyaku Co., Ltd., Novartis Pharma K.K., Pfizer Japan Inc., Janssen Pharmaceutical K.K., Daiichi Sankyo Co., Ltd., and Mochida Pharmaceutical Co., Ltd. (none of which are related to the submitted work). The other authors declare that they have no conflicts of interest.

Publisher's Note Springer Nature remains neutral with regard to jurisdictional claims in published maps and institutional affiliations.

The original article can be found online at https://doi.org/10.1007/ s12185-021-03228-1.

Hirohisa Nakamae

hirohisa@med.osaka-cu.ac.jp

Hematology, Graduate School of Medicine, Osaka City University, 1-4-3 Asahi-machi, Abeno-ku, Osaka 545-8585, Japan 